\title{
Automatización de un Reactor Tipo Batch para la obtención de Biodiesel a partir de aceite reciclado
}

DOI: https://doi.org/10.33262/ap.v3i3.68

\begin{abstract}
(c) ()ㅇㅇㅣ BY NC SA

\section{Automation of a Batch Reactor to obtain Biodiesel from recycled oil}

Jonatahn Paúl Campos Castelo. ${ }^{1}$, Nelly Jacqueline Coba Castillo. ${ }^{2}$, Irma Catalina Villa Escudero. ${ }^{3} \&$ Adrian Alejandro Aldaz Vargas. ${ }^{4}$
\end{abstract}

\begin{abstract}
.
Introduction: Today, thanks to the innovation and development of new technologies, it is possible to automate industrial processes which help to improve the production process and generate a competitive market. Commercial and industrial companies in Ecuador have automated systems that are capable of facilitating work and obtaining quality products, reducing costs and increasing production. Methodology: For the development and execution of this project of Automation of a batch type reactor to obtain biodiesel in the Laboratory of Industrial Processes of the Faculty of Sciences, information on the production process, the reagents used, materials and necessary equipment. The following methods were applied: inductive, deductive and experimental. Results: The automation allowed the reduction of the time in the decantation phase by $81.25 \%$; which helps the production time to also be reduced by $65.22 \%$ compared to 11 hours and 30 minutes when the process that was purely manual according to the tests obtained in the automation
\end{abstract}

\footnotetext{
${ }^{1}$ Instituto Superior Tecnológico "Juan de Velasco", Riobamba, Ecuador, paulcamposjuandevelasco@gmail.com, ORCID: 0000-0002-8243-451X

2 Instituto Superior Tecnológico "Juan de Velasco", Riobamba, Ecuador, nellyjacquelinejc@gmail.com, ORCID: 0000-0002-1093-3977

3 Instituto Superior Tecnológico "Juan de Velasco", Riobamba, Ecuador, irmacatalina81@gmail.com, ORCID: 0000-0002-8057-6020

${ }^{4}$ Universidad Nacional de Chimborazo, Riobamba, Ecuador, aaldaz@unach.edu.ec, ORCID: 0000-00016290-0539
} 
presents an improvement in the quality of the biodiesel. Conclusion: The performance of the automated reactor is notably increased by $287.5 \%$. There is an operating equipment with low current consumption, a reduction in the amount of water used to wash the biodiesel and it has a constant agitation system. The volume yield of the biodiesel obtained is highly dependent on the quality of the recycled cooking oil and the purity of the methanol.

Keywords: Process automation, biodiesel reactor, biodiesel production, programmable logic controller, recycled oil.

\section{Resumen.}

Introducción: Hoy en día gracias a la innovación y desarrollo de nuevas tecnologías es posible la automatización de procesos industriales los cuales ayudan a mejorar el proceso de producción y generar un mercado competitivo. Las empresas comerciales e industriales del Ecuador cuentan con sistemas automatizados que son capaces de facilitar el trabajo y obtener productos de calidad reduciendo costos e incrementando la producción. Metodología: Para el desarrollo y ejecución de este proyecto de Automatización de un reactor tipo batch para la obtención de biodiesel en el Laboratorio de Procesos Industriales de la Facultad de Ciencias, se revisó información sobre el proceso de producción, los reactivos que se emplean, materiales y equipos necesarios. Se aplicaron los siguientes métodos: inductivo, deductivo y experimental. Resultados: La automatización permitió la reducción del tiempo en la fase de decantación en un 81,25\%; lo que ayuda a que el tiempo de producción también se reduzca en un 65,22\% comparado a las 11 horas y 30 minutos cuando el proceso que era netamente manual de acuerdo con las pruebas obtenidas en la automatización se presenta una mejora en la calidad del biodiesel. Conclusión: El rendimiento del reactor automatizado se incrementa notablemente en $287,5 \%$. Se tiene un equipo operativo con bajo consumo de corriente, reducción en la cantidad de agua empleada para el lavado del biodiesel y posee un sistema de agitación constante. El rendimiento en volumen del biodiesel obtenido depende en gran medida de la calidad del aceite de cocina reciclado y la pureza del metanol.

Palabras claves: Automatización de procesos, reactor de biodiesel, producción de biodiesel, controlador lógico programable, aceite reciclado.

\section{Introducción.}

A través de los siglos el ser humano ha creado un conjunto de herramientas y máquinas de acuerdo con las necesidades que se presentaban, de ahí nació la revolución industrial con el fin de optimizar el trabajo a través de la mecanización. Las primeras máquinas remplazaban el esfuerzo del ser humano, posteriormente con el uso de nuevas tecnologías 
estas máquinas fueron capaces de realizar un conjunto de tareas sustituyendo el trabajo del hombre.

La automatización industrial nació con la evolución de la electrónica y las computadoras que son dos herramientas necesarias para un proceso automatizado que permite controlar y operar adecuadamente un sistema, es por ello que los beneficios que proporciona un sistema automatizado son múltiples ya que cada día surgen nuevas aplicaciones y nuevos procesos.

Hoy en día gracias a la innovación y desarrollo de nuevas tecnologías es posible la automatización de procesos industriales los cuales ayudan a mejorar el proceso de producción y generar un mercado competitivo. Las empresas comerciales e industriales del Ecuador cuentan con sistemas automatizados que son capaces de facilitar el trabajo y obtener productos de calidad reduciendo costos e incrementando la producción.

Para la realización de esta investigación se tomó como punto de referencia el trabajo realizado por Espinoza y Palmay (2009) quienes realizaron el ' Diseño y construcción de un reactor batch para la obtención por transesterificación de biodiesel a partir de aceite de cocina reciclado" este procedimiento se lo realizaba de manera manual es por ello que se planteó una alternativa la automatización de los procesos para la producción del biodiesel los mismos que permitieron mejorar la calidad del producto y la reducción del tiempo de producción.

\section{Metodologia.}

Para el desarrollo y ejecución de este proyecto de Automatización de un reactor tipo batch para la obtención de biodiesel en el Laboratorio de Procesos Industriales de la Facultad de Ciencias, se revisó información sobre el proceso de producción, los reactivos que se emplean, materiales y equipos necesarios. Se aplicaron los siguientes métodos: inductivo, deductivo y experimental.

Inductivo: Ayudó a determinar y definir parámetros como temperatura, velocidad de agitación, concentración y pureza de los compuestos químicos.

Deductivo: La existencia de información relevante sobre la producción de biodiesel aportó significativamente al desarrollo de este proyecto, permitió conocer las etapas o fases de producción, los reactivos que pueden emplearse.

Experimental: Permitió la corrección de errores y fallas que se presentaron en la maniobra y operación del reactor. Con este método se procedió a la ubicación y calibración de sensores, depuración en los tiempos de reacción y velocidad de agitación según la etapa, referente a la parte eléctrica y electrónica; mientras que en la parte química permite determinar las cantidades exactas de reactivos y el manejo adecuado de los mismos. 
Tabla 1-2: Fases del proceso de obtención del biodiesel

\begin{tabular}{|c|c|}
\hline Fase & Descripción \\
\hline $\begin{array}{l}\text { Eliminación de } \\
\text { impurezas }\end{array}$ & $\begin{array}{l}\text { Consiste en filtrar el aceite reciclado de forma manual y después, } \\
\text { someterlo a una temperatura de } 100{ }^{\circ} \mathrm{C} \text { para eliminar el agua que } \\
\text { podría contener. }\end{array}$ \\
\hline Transesterificación & $\begin{array}{l}\text { Se mezcla el aceite, el alcohol metílico en proporción del } 20 \% \text { del } \\
\text { volumen de aceite y } 3.5 \text { gramos de hidróxido de sodio por cada } \\
\text { litro de aceite; esta mezcla se calienta a } 58^{\circ} \mathrm{C} \text { con una agitación } \\
\text { rápida al inicio y lenta hacia el final de la reacción. }\end{array}$ \\
\hline Decantación & $\begin{array}{l}\text { La mezcla debe reposar al menos } 8 \text { horas para poder separar la } \\
\text { glicerina y el biodiesel o en su defecto se puede dejar la mezcla } \\
\text { en reposo, después de la reacción, pero manteniendo una } \\
\text { temperatura sobre los } 38^{\circ} \mathrm{C} \text { por al menos una hora lo cual permite } \\
\text { la reducción del tiempo en esta fase. }\end{array}$ \\
\hline Lavado & $\begin{array}{l}\text { Consiste en separar los jabones del biocombustible lavándolo con } \\
\text { agua a una proporción del } 1 / 3 \text { del volumen de aceite cargado y la } \\
\text { agitación se produce a bajas velocidades, el agua ingresa desde } \\
\text { uno de los tanques secundarios, posteriormente la mezcla debe } \\
\text { permanecer en reposo para separar los líquidos. }\end{array}$ \\
\hline Secado & $\begin{array}{l}\text { El biocombustible obtenido es calentado nuevamente a } 100^{\circ} \mathrm{C} \\
\text { para eliminar el agua presente. Finalmente se obtiene el biodiesel. }\end{array}$ \\
\hline
\end{tabular}

Fuente: Elaboración propia

Limpieza del reactor y tuberías.

El reactor permanecía mucho tiempo sin utilizarse por lo que se había acumulado polvo y había residuos de biodiesel y glicerina en la cámara de ebullición, en los recipientes de carga y en la tubería de descarga. Se procede a la limpieza integral, desarmando todo el reactor, utilizando jabón arranca grasa y agua caliente para la eliminación de la glicerina acumulada.

Vaciado del vapor condensado, instalación de una trampa de vapor.

El reactor no tenía incorporado una trampa de vapor que permita la descarga del condensado generado durante los procesos en las líneas que transportan vapor, por lo que había gran cantidad de agua y es estrictamente necesario desalojarla de la cámara de calefacción, fue posible gracias a una llave de desfogue instalada en la parte inferior del reactor. 


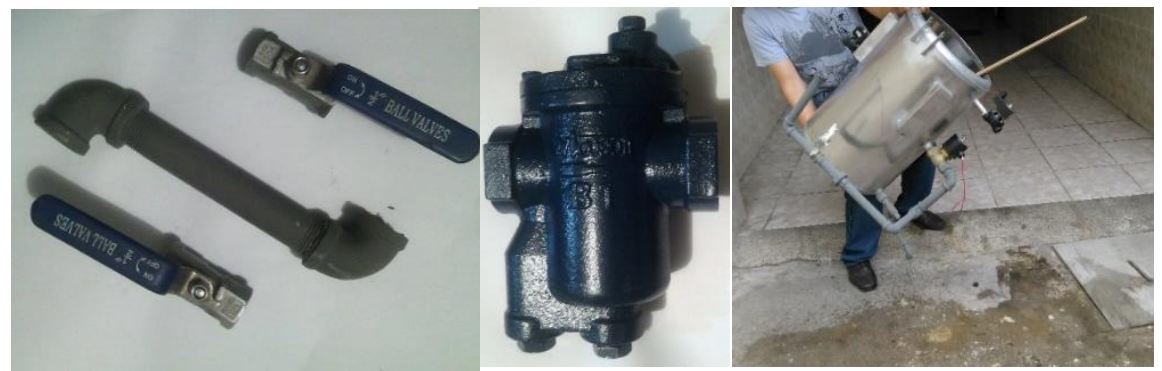

Figura 1-2 Vaciado del condesado, trampa de vapor Fuente: Elaboración propia

Se instaló la trampa de vapor, la misma que presenta las siguientes características:

Tabla 2-2: Características de la trampa de vapor

\begin{tabular}{ll}
\hline Tipo de Trampa & Mecánica \\
\hline Marca/Modelo & Nicoson/B1 \\
Conexión & 1 \\
Presión Máxima & $14 \mathrm{~kg} / \mathrm{cm}^{2}$ \\
\hline
\end{tabular}

Fuente: Elaboración propia

Incorporación de la estructura para el gabinete eléctrico

Se elimina la estructura del pequeño gabinete antiguo para colocar uno nuevo de las dimensiones necesarias que pueda abarcar en su interior todos los elementos necesarios. Se instala en una nueva ubicación donde no interfiera con la máquina y resulte fácil de acceder y operar.

Se realiza un soporte para la bomba de succión que se emplea en cargar el aceite o biodiesel al reactor, se instala en la parte inferior junto al recipiente de descarga.

Colocación de un nuevo tanque secundario, sensores de nivel y electroválvulas

Se coloca un nuevo tanque secundario de acero inoxidable para contener uno de los reactivos ya que el anterior era muy pequeño y no cabía la cantidad mínima necesaria para la reacción; estos sensores de nivel tipo flotador se emplean para detectar si los tanques secundarios contienen la cantidad mínima de metóxido o agua para dar paso a la fase de transesterificación o lavado del biodiesel respectivamente.

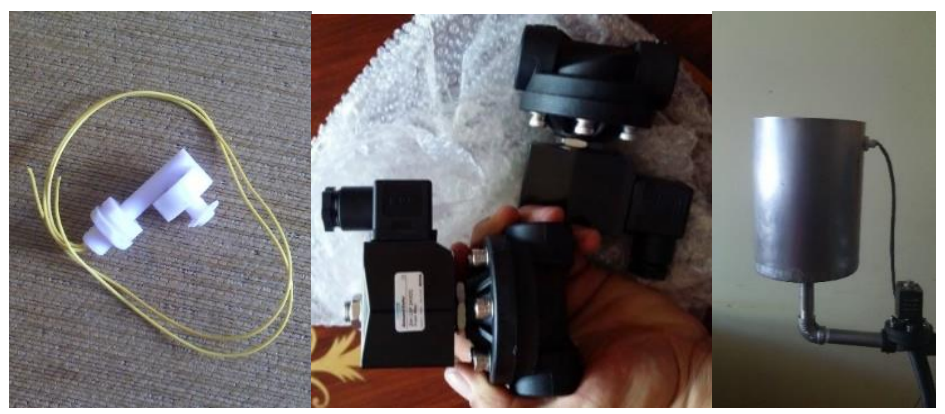

Figura 2-2 Arreglos en el reactor

Fuente: Elaboración propia 
Las electroválvulas instaladas presentan los siguientes datos:

Tabla 3-2: Características de las electroválvulas de CC.

\begin{tabular}{ll}
\hline Alimentación & $24 \mathrm{VDC}$ \\
Potencia de Consumo & $20 \mathrm{~W}$ \\
Tipo & Normalmente Cerrada \\
Presión de Operación & $0-8 \mathrm{Bar}$ \\
Conexión & $1 / 2$ “ \\
Protección & IP 65 \\
\hline
\end{tabular}

Fuente: Elaboración propia

Construcción de las aspas para el motor agitador

Se empleo un nuevo motor y un nuevo brazo de paletas de acero inoxidable y sujetándolo a la tapa del reactor. Se colocó un motor de 24 VDC con reducción incorporado para generar torque suficiente y mezclar los líquidos sin generar vórtices. Se realiza ese diseño de aspas porque su implementación es sencilla y económica.

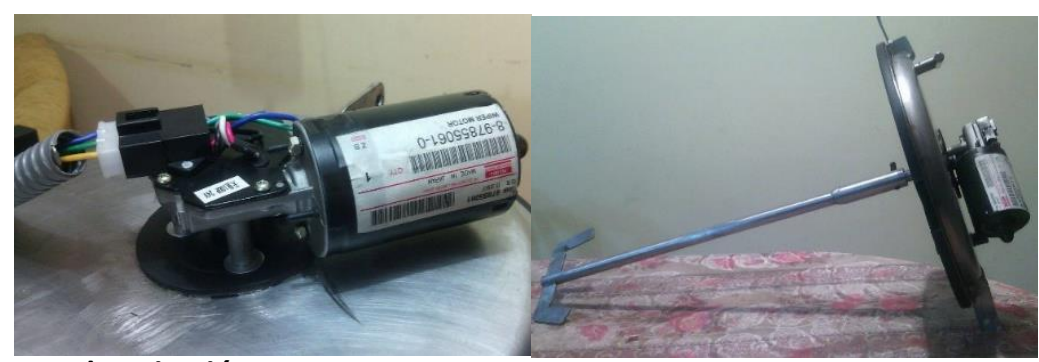

Figura 3-2. Sistema de agitación

Fuente: Elaboración propia

Selección de dispositivos de control y sensores

Los pulsadores son importantes para la operación y control de la máquina, consta de tres botones: botón de inicio para arrancar el proceso; botón de paro de emergencia para cuando se precise detener el proceso por cuestiones de seguridad o debido a algún funcionamiento anormal y pulsador de Reset. Las luces piloto funcionan como indicadores del estado del reactor, la luz de color verde indica un correcto funcionamiento y operación dentro de los parámetros establecidos, la luz de color rojo indica que la máquina se encuentra en estado de PARO/STOP y luz de color amarillo indica que se ha reseteado el equipo.

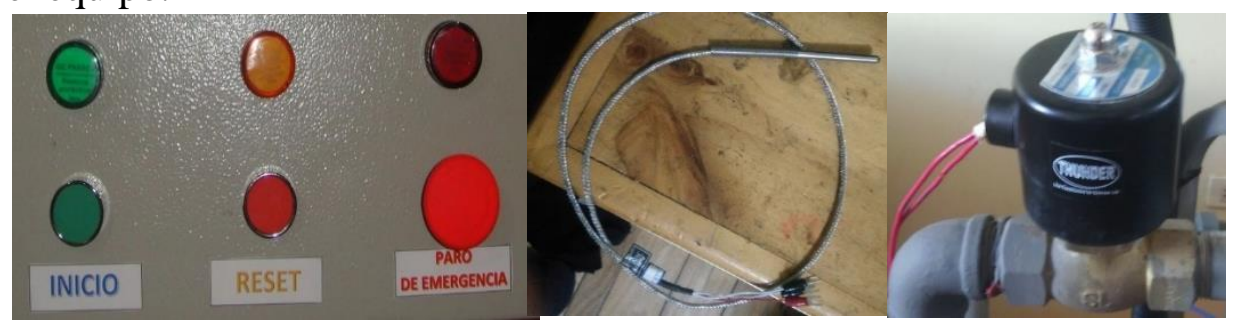

Figura 4-2 Pulsadores, luces piloto, pt100 y electroválvula

Fuente: Elaboración propia 
Sensor de temperatura PT100

El sensor de temperatura se instaló en el fondo del reactor y permite conocer la temperatura en la cámara de calefacción durante cada fase; según el valor leído y las condiciones programadas en el PLC se realiza el control de la apertura o cierre de la electroválvula de paso de vapor.

Se utilizó el sensor PT100 de 3 hilos porque el rango de medida habitual está comprendido entre $-100^{\circ} \mathrm{C}$ y $350^{\circ} \mathrm{C}$. La electroválvula para el paso de vapor se selecciona por su temperatura de operación $\left(-5\right.$ a $\left.180^{\circ} \mathrm{C}\right)$ y presión de trabajo $\left(0,5\right.$ a $\left.15 \mathrm{~kg} / \mathrm{cm}^{2}\right)$

Selección de PLC y módulo de funciones especiales (Board BD)

La selección del Controlador Lógico Programable se realiza en función del número de entradas y salidas a emplearse y dependiendo si éstas son digitales o analógicas.

En la siguiente tabla se detalla el número de entradas requeridas.

Tabla 4-2: Descripción de entradas al PLC

\begin{tabular}{ccc}
\hline Descripción de Entrada & Tipo de Señal & Cantidad \\
\hline Sensor temperatura PT100 & Analógica & 1 \\
Pulsador inicio & Digital & 1 \\
Pulsador reset & Digital & 1 \\
Pulsador paro emergencia & Digital & 1 \\
Sensores de nivel & Digital & 2 \\
TOTAL & & $\mathbf{6}$
\end{tabular}

Fuente: Elaboración propia

La siguiente tabla presenta el detalle y número de salidas a manejarse.

Tabla 5-2: Descripción de salidas del PLC

\begin{tabular}{lcc}
\hline Descripción de Salida & Tipo de Señal & Cantidad \\
\hline Luz piloto de inicio & Digital & 1 \\
Luz piloto reset & Digital & 1 \\
Luz piloto paro & Digital & 1 \\
Electroválvulas & Digital & 3 \\
Motor agitador & Digital & 2 \\
Bomba de succión & Digital & 1 \\
TOTAL & & 9
\end{tabular}

Fuente: Elaboración propia

Por lo tanto, se requiere un PLC que permita el manejo de 6 entradas, una de ellas de tipo analógica y 9 salidas digitales. El controlador seleccionado debe permitir la incorporación 
de un módulo para el manejo de señales analógicas provenientes del sensor de temperatura PT100.

Selección y características del módulo de funciones especiales (Board BD)

Para el manejo de la señal proporcionada por el PT100 se requiere de una tarjeta de funciones especiales XC-2AD2PT que es compatible con el PLC Xinje XC3-24R-E y PLCs de la serie XC, esta tarjeta posee 4 canales, de los cuales 2 son canales directos para la conexión de sensores PT100 y los otros 2 canales para señales analógicas que se pueden configurar en rangos de $0-5 \mathrm{v}$ o $0-10 \mathrm{v}$.

\section{Características del PLC seleccionado}

El PLC seleccionado es el modelo XC3-24R-E de la marca Xinje que se muestra en la Figura 7-2 y presenta las características que se aprecia en la Tabla 6-2.
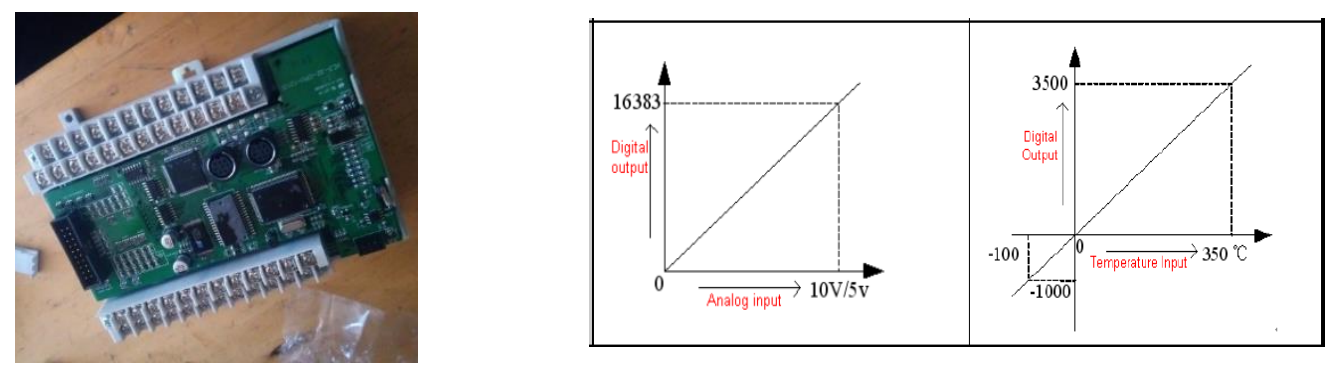

Figura 7-2 PLC Xinje XC3-24R-E y comportamiento de las señales analógicas BD

Fuente: Elaboración propia

Tabla 6-2: Características del PLC Xinje XC3-24R-E

\begin{tabular}{lcl}
\hline \multicolumn{1}{c}{ Descripción } & Cantidad & \multicolumn{1}{c}{ Tipo } \\
\hline Voltaje de alimentación & 1 & 110/220 VAC \\
Fuente interna & 1 & 24 VDC \\
Entradas & 14 & Digitales 24 VDC \\
COM Entradas & 2 & 0 VDC \\
Salidas a relé & 10 & Digitales \\
COM Salidas & 3 & Fuentes variables \\
$\begin{array}{l}\text { Puertos de comunicación } \\
\text { expansión }\end{array}$ & 2 & COM1 (RS-232), COM2 \\
Módulos & 7 & (RS-232/RS-485) \\
permitidos funciones & 1 & Varios modelos \\
Tarjeta de & & \\
especiales (Board BD) & &
\end{tabular}

Fuente: Elaboración propia 
El incremento de la resistencia del PT 100 no es lineal, pero si creciente, entonces la tarjeta asigna un valor proporcional y lineal de temperatura según incrementa la resistencia.

La Board BD se instala directamente sobre el PLC y la configuración se realiza con el software XC Series Program Tool.

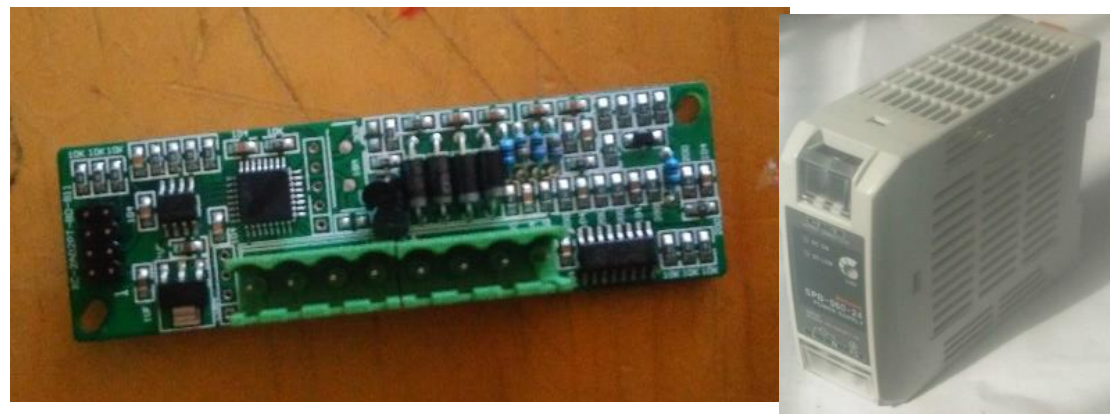

Figura 6-2 Tarjeta de funciones especiales XC-2AD2PT y Fuente Autonics 24VDC

Fuente: Elaboración propia

\section{Selección de la fuente externa}

La fuente empleada tiene las siguientes características. marca Autonics, modelo SPB060-24, proporciona 24 VDC y 2.5 A, puede conectarse a 110 o 220 VAC. Se utilizó para accionar las electroválvulas empleadas en los recipientes de metóxido y agua; para alimentar el motor agitador, sensores; luces piloto y energizar las bobinas.

Diseño e implementación del gabinete eléctrico

En el gabinete eléctrico se instalan todos los elementos de control, tiene las siguientes características: marca Beaucoup, mide 400 x 400 x $200 \mathrm{~mm}$, cuerpo y doble fondo fabricado en acero laminado en frío.

Conexiones, dimensionamiento de conductores y protecciones

- PT100: Se utilizó alambre de cobre como extensión para el PT100 de 1,5 m y se escogió el canal 3 para conectarlo.
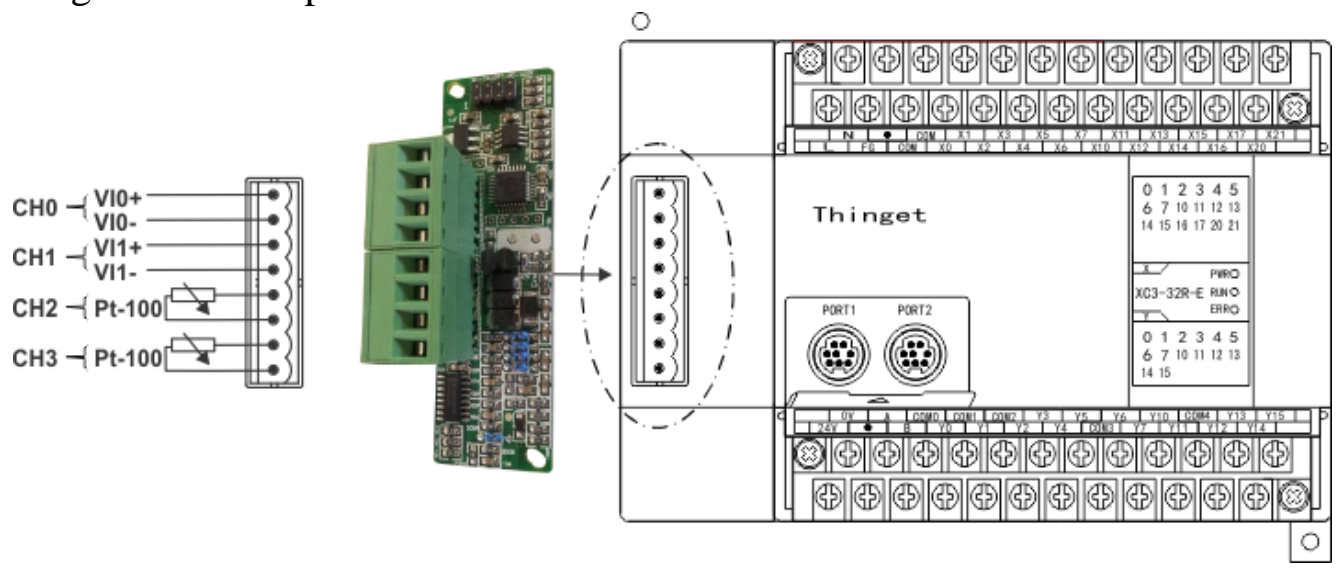

Figura -2 Conexión PT100

Fuente: http://www.sah.rs/PLC\%20and\%20HMI/XC-BD_eng.html 
- Bomba de agua:

La bomba de agua es la carga que mayor consumo de potencia tiene. Para determinar el calibre del conductor que se emplea para la motobomba, se tiene los siguientes datos:

Voltaje nominal: $110 \mathrm{VAC}$

Corriente nominal: 3,4 A

Longitud: $2 \mathrm{~m}$

Se calcula la caída de tensión en un circuito monofásico, debe ser menor al 5\%.

$$
\begin{gathered}
\Delta V=2 I \operatorname{Re} L \\
\Delta V=2(3,4)(0,0134)(2) \\
\Delta V=0,182 \%
\end{gathered}
$$

Se determina la sección del conductor eléctrico

\begin{tabular}{|c|c|c|c|c|c|c|c|c|c|}
\hline $\begin{array}{l}\text { CALIBRE } \\
\text { AWG } \\
\text { ó MCM }\end{array}$ & $\begin{array}{c}\text { SECCION } \\
\mathrm{mm}^{2}\end{array}$ & $\begin{array}{l}\text { FORMACION } \\
\text { No. de hilos } \\
\text { por } \\
\text { diámetro } \\
\text { mm. }\end{array}$ & $\begin{array}{c}\text { ESPESOR } \\
\text { AISLAMIENTO } \\
\mathrm{mm} .\end{array}$ & $\begin{array}{c}\text { ESPESOR } \\
\text { CHAQUETA } \\
\text { mm. }\end{array}$ & $\begin{array}{c}\text { DIAMEIRO } \\
\text { EXIERIOR } \\
\mathrm{mm} .\end{array}$ & $\begin{array}{l}\text { PESO } \\
\text { TOTAL } \\
\mathrm{Kg} / \mathrm{Km}\end{array}$ & $\begin{array}{c}\text { CAPAC. DE } \\
\text { CORRIENTE } \\
\text { para } 1 \\
\text { conductor } \\
\text { al aire libre } \\
\text { Amp. }\end{array}$ & $\begin{array}{c}\text { CAPAC. DE } \\
\text { CORRIENTE } \\
\text { para } 3 \\
\text { conductores } \\
\text { en conduit } \\
\text { Amp. }\end{array}$ & \begin{tabular}{|l} 
TIPO \\
CABLE
\end{tabular} \\
\hline 20 & 0,519 & $1 \times 0.813$ & 0,38 & 0,10 & 1,77 & 7,07 & 15 & 10 & TFN \\
\hline 18 & 0,823 & $1 \times 1,02$ & 0,38 & 0,10 & 1,98 & 10,94 & 15 & 10 & TFN \\
\hline 16 & 1,31 & $1 \times 1,29$ & 0,38 & 0,10 & 2,25 & 16,48 & 20 & 15 & TFN \\
\hline 14 & 2,08 & $1 \times 1,63$ & 0,38 & 0,10 & 2,59 & 23,17 & 35 & 25 & THHN \\
\hline 12 & 3,31 & $1 \times 2,05$ & 0,38 & 0,10 & 3,01 & 34,16 & 40 & 30 & THHN \\
\hline 10 & 5,26 & $1 \times 2,59$ & 0,51 & 0,10 & 3,81 & 55,04 & 55 & 40 & THHN \\
\hline 8 & 8,34 & $1 \times 3,26$ & 0,76 & 0,13 & 5,04 & 91,22 & 80 & 55 & THHN \\
\hline 16 & 1,31 & $19 \times 0,30$ & 0,38 & 0,10 & 2,46 & 17,95 & 20 & 15 & TFN \\
\hline 14 & 2,08 & $19 \times 0,38$ & 0,38 & 0,10 & 2,86 & 23,80 & 35 & 25 & THHN \\
\hline 12 & 3,31 & $19 \times 0,47$ & 0,38 & 0,10 & 3,31 & 35,70 & 40 & 30 & THHN \\
\hline 10 & 5,26 & $19 \times 0,60$ & 0,51 & 0,10 & 4,22 & 56,20 & 55 & 40 & THHN \\
\hline 8 & 8,37 & $7 \times 1,23$ & 0,76 & 0,13 & 5,47 & 93,70 & 80 & 55 & THHN \\
\hline 6 & 13,30 & $7 \times 1,55$ & 0,76 & 0,13 & 6,43 & 141,30 & 105 & 75 & THHN \\
\hline 4 & 21,15 & $7 \times 1,96$ & 1,02 & 0,15 & 8,22 & 227,60 & 140 & 95 & THHN \\
\hline 2 & 33,62 & $7 \times 2,47$ & 1,02 & 0,15 & 9,75 & 348,10 & 190 & 130 & THHN \\
\hline 1 & 42,36 & $7 \times 2,78$ & 1,27 & 0,18 & 11,24 & 446,20 & 220 & 150 & THHN \\
\hline
\end{tabular}

$$
\begin{gathered}
S e=\frac{2 * R e * I * L}{V p} \\
S e=\frac{2 *(0,0134) * 3,4 * 2}{0,182 * 110} \\
S e=0,091 \mathrm{~mm}^{2} \\
D=2 * \sqrt{\frac{S e}{\pi}} \\
D=0,34 \mathrm{~mm}
\end{gathered}
$$

El diámetro determina que el calibre de cable a utilizarse es el 20 AWG, según la tabla de la Figura 12-2. 
La siguiente tabla muestra el detalle de las cargas instaladas, cuyo consumo de potencia es muy bajo y las distancias son cortas. Por lo tanto, para su cableado se utiliza cables de calibre 18 AWG.

La Figura 9-2 muestra el diagrama de conexiones de las entradas y salidas en el Controlador Lógico Programable.

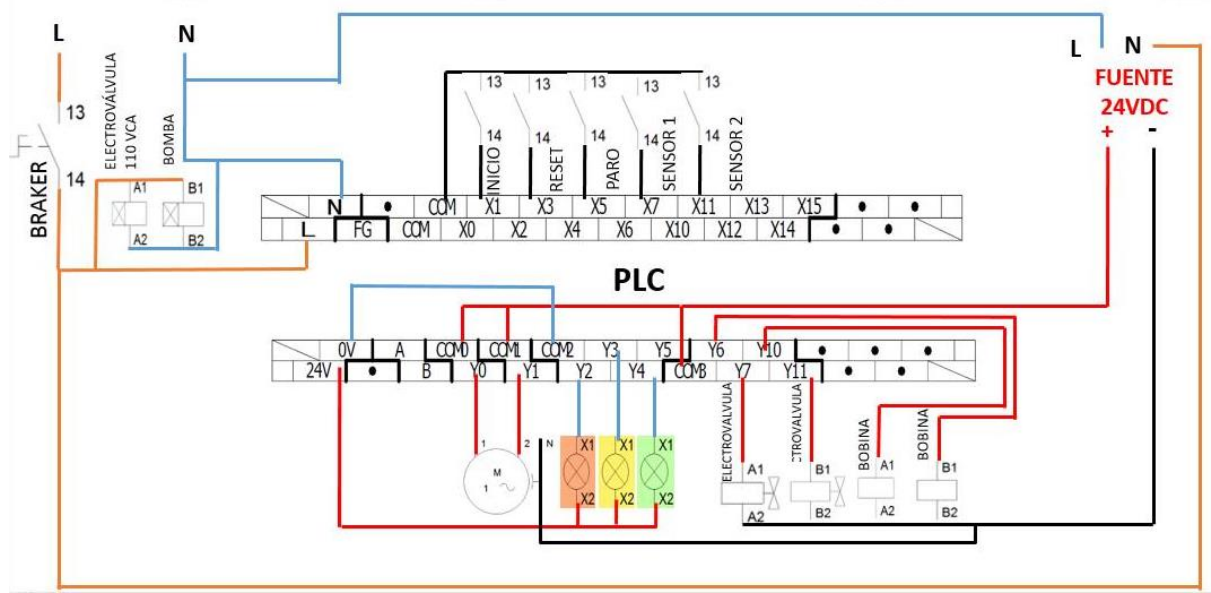

Figura 9-2 Diagrama de conexiones del PLC

Fuente: Elaboración propia

Para las cargas que requieren de una alimentación de 24 VDC se realiza las conexiones con cable flexible número 18 AWG, las conexiones de AC para alimentación del PLC y la fuente se realiza con cable flexible número 16 AWG, y finalmente para la alimentación desde la toma de 110 VAC al gabinete se emplea conductor flexible tipo gemelo número 14 AWG.

Tabla 8-2: Elementos del gabinete

\begin{tabular}{lll}
\hline \multicolumn{1}{c}{ Cantidad } & \multicolumn{1}{c}{ Descripción } & \multicolumn{1}{c}{ Ubicación } \\
\hline 1 & Controlador Lógico Programable & Interior del gabinete \\
1 & Breaker de 10 A & Interior del gabinete \\
1 & Fuente de 24 VDC / 2,5 A & Interior del gabinete \\
2 & Relé 24 VDC, 8 pines con base & Interior del gabinete \\
14 & Borneras & Interior del gabinete \\
1 & Pantalla de interfaz & Compuerta del gabinete \\
3 & Pulsadores & Compuerta del gabinete \\
3 & Luces piloto & Compuerta del gabinete \\
\hline
\end{tabular}

Fuente: Elaboración propia

Se emplearon taladros y disco de corte para realizar las perforaciones en las que se colocaron los pulsadores, luces piloto y la pantalla de interfaz. 

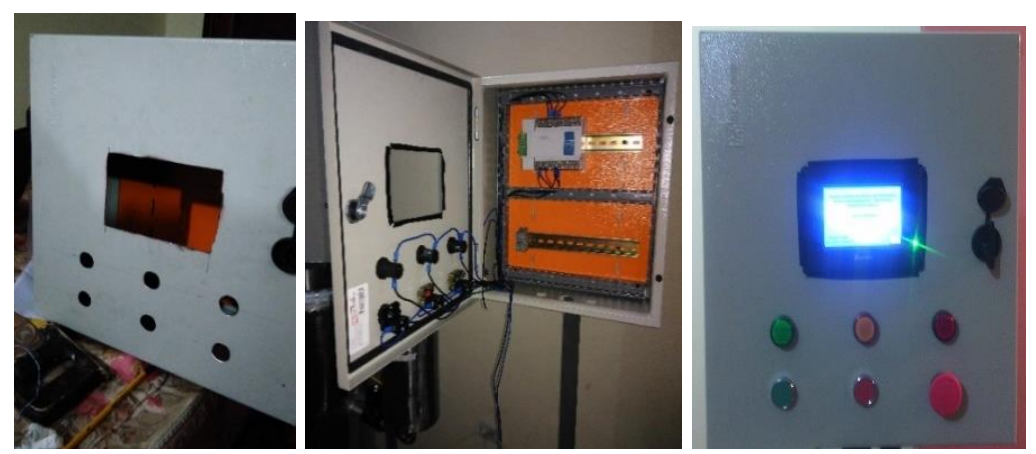

Figura 10-2 Gabinete eléctrico

Fuente: Elaboración propia

\section{Diagramas GRAFCET}

El GRAFCET es una herramienta que permite hacer un bosquejo de la programación que se lleva a cabo, es como una guía del funcionamiento general del reactor que describe cómo se desarrollaría el proceso de automatización. Aunque se emplea un GRAFCET por cada etapa del proceso, en realidad el programa implementado es uno solo. Las letras X, Y, M, T; indican entradas, salidas, memorias y temporizadores respectivamente.
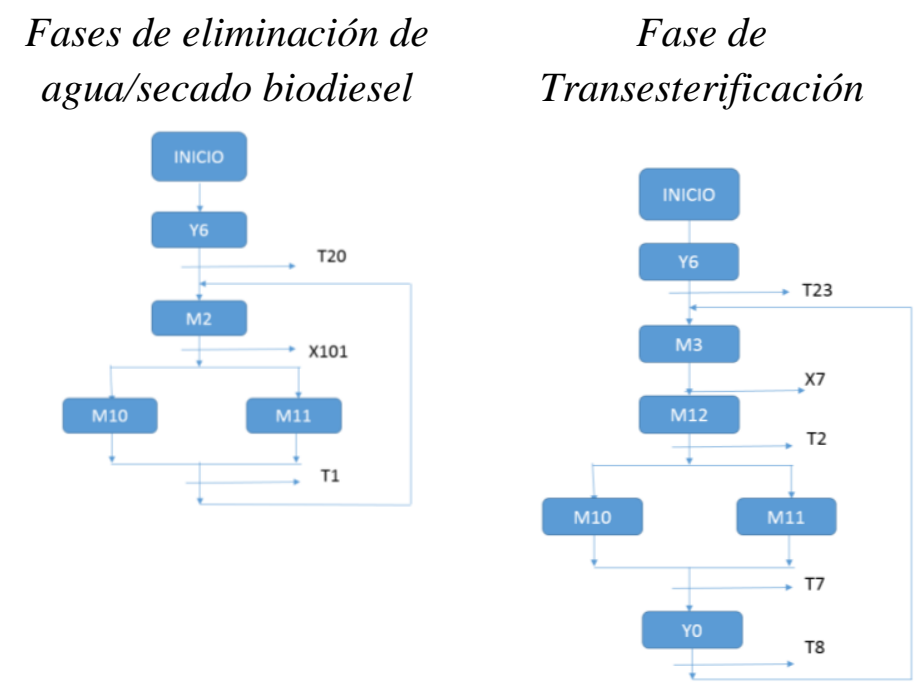

Fase de lavado del biodiesel

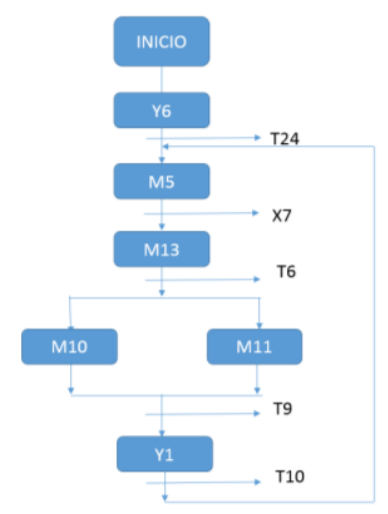

Figura 11-2 Diagramas GRAFCET según etapas

Fuente: Elaboración propia

\section{Interfaz Hombre Máquina (HMI)}

La pantalla de interfaz seleccionada para el control y monitoreo del proceso de obtención de biodiesel tiene las siguientes características: marca TouchWin, modelo TG465-UT, pantalla táctil, voltaje de operación de $24 \mathrm{VDC}$, resolución $480 * 272$ pixeles, 4.3 " de tamaño de la pantalla.

Pantallas: principal y de selección

Al encender el equipo, aparece una pantalla de presentación, al presionar sobre la opción "Iniciar" se accede a otra ventana que contiene botones cuya selección permite que el proceso se ejecute de forma manual o automática. 


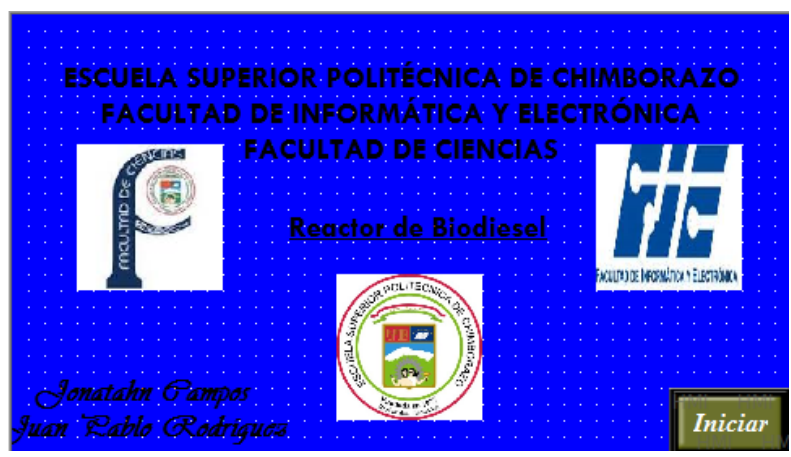

Figura 12-2 Pantalla de presentación

Fuente: Elaboración propia

La siguiente pantalla permite seleccionar el modo de operación del equipo, manual o automático. En el caso de seleccionarse el modo automático, la carga de aceite es máximo 15 litros y el caldero debe tener una presión mínima de 40 PSI.

En el modo automático se presenta la pantalla siguiente, en la que consta cada fase de la producción del biodiesel, al seleccionar cualquiera de ellas, se presenta una nueva pantalla a través de la cual se da inicio a la fase seleccionada.

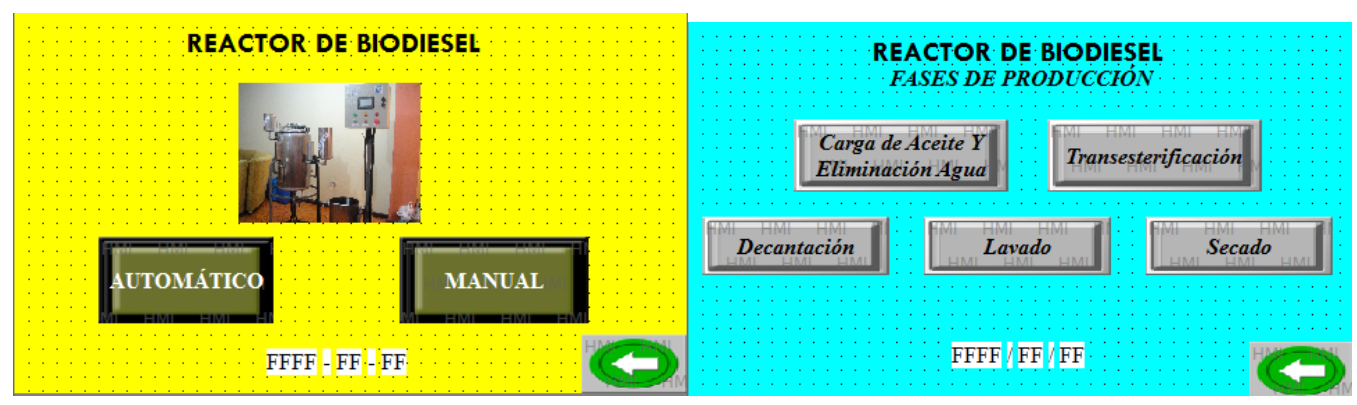

Figura 13-2 Pantalla modo automático Fuente: Elaboración propia

En el modo manual se tiene la pantalla de la siguiente figura, este modo permite la maniobra del equipo cuando las cargas de aceite son menores o mayores de 15 litros o cuando se requiera utilizar el reactor en otros proyectos no necesariamente relacionados con la producción de biodiesel, empleándose otros compuestos y reactivos químicos en volúmenes diferentes.
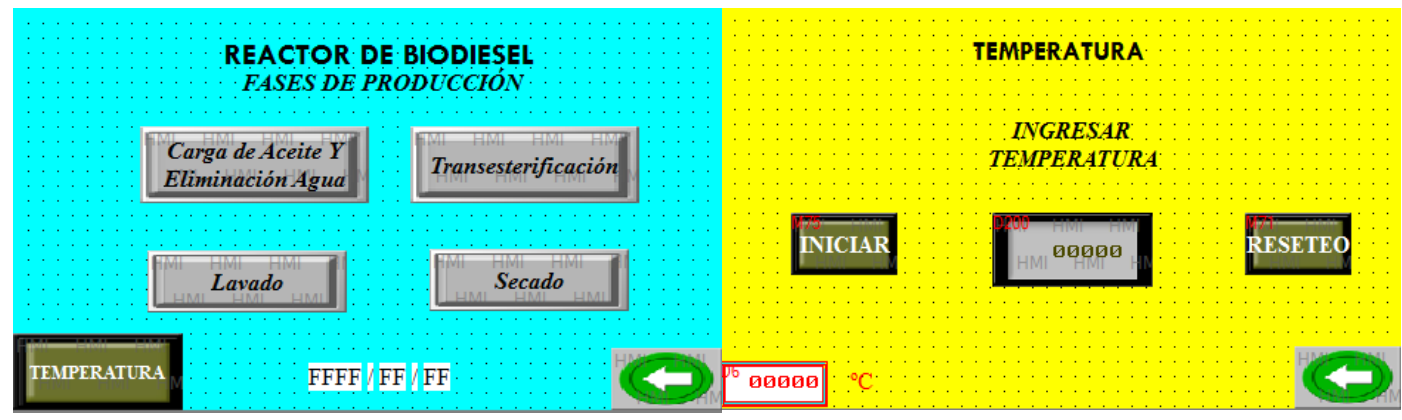

Figura 14-2 Pantalla modo manual

Fuente: Elaboración propia 
Al seleccionar en la opción Temperatura, una nueva ventana indica el ingreso de un valor de temperatura, la misma controla que la electroválvula permita o corte el paso del vapor hacia la cámara de calefacción. En función del valor ingresado la electroválvula se maneja con una tolerancia de $\pm 2^{\circ} \mathrm{C}$. Dentro del modo de operación manual se tiene una pantalla para cada fase, desde las cuales se enciende o apaga cualquiera de los actuadores.
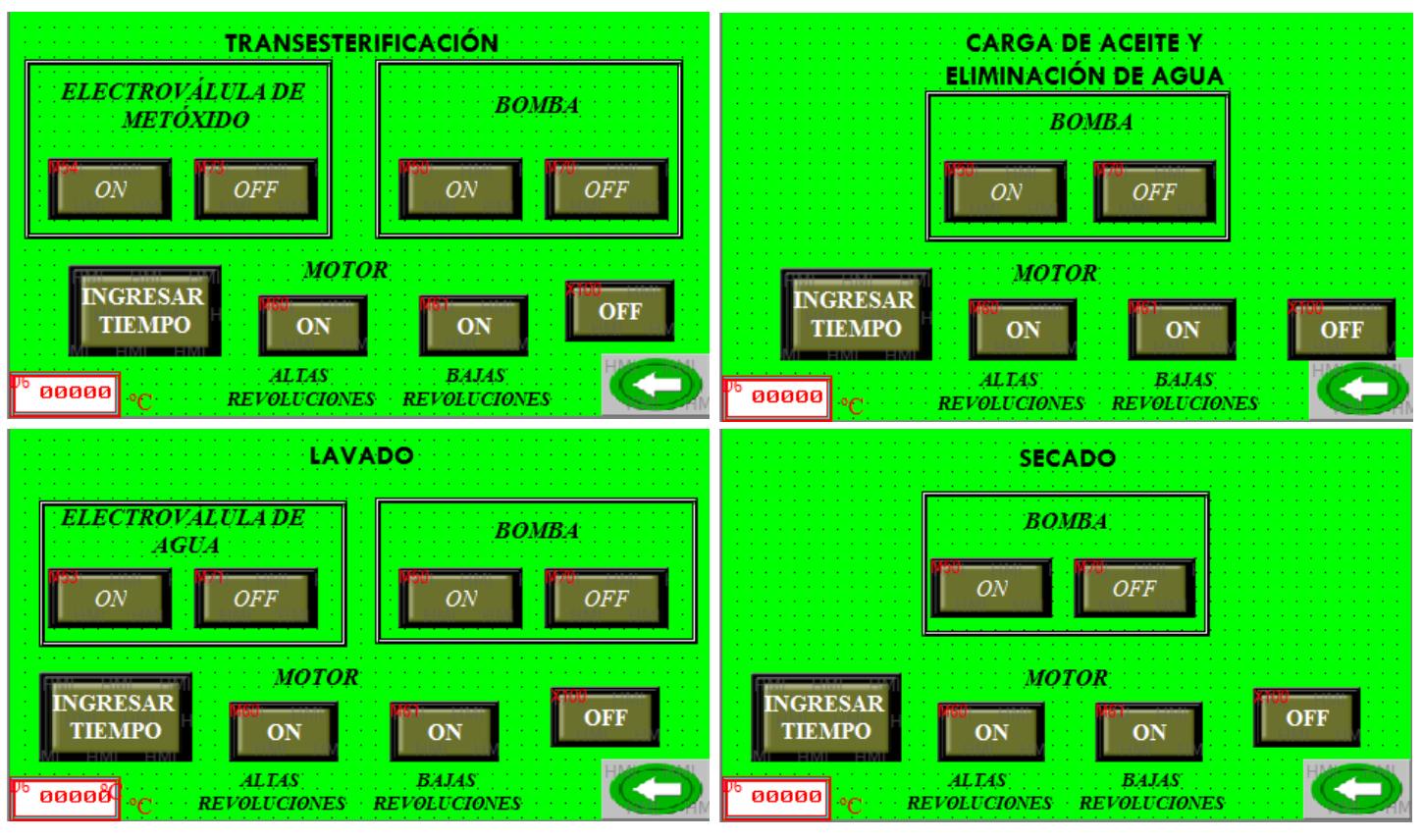

Figura 15-2 Pantallas modo manual para cada fase Fuente: Elaboración propia

Para accionar el sistema de agitación por un tiempo determinado, se presiona sobre el botón Ingresar Tiempo, que redirecciona a otra pantalla en la cual se puede ingresar el tiempo durante el cual se mantendrá encendido el motor sea en velocidad rápida o velocidad baja, para ingresar un nuevo valor hay que pulsar en Reinicio Contador.

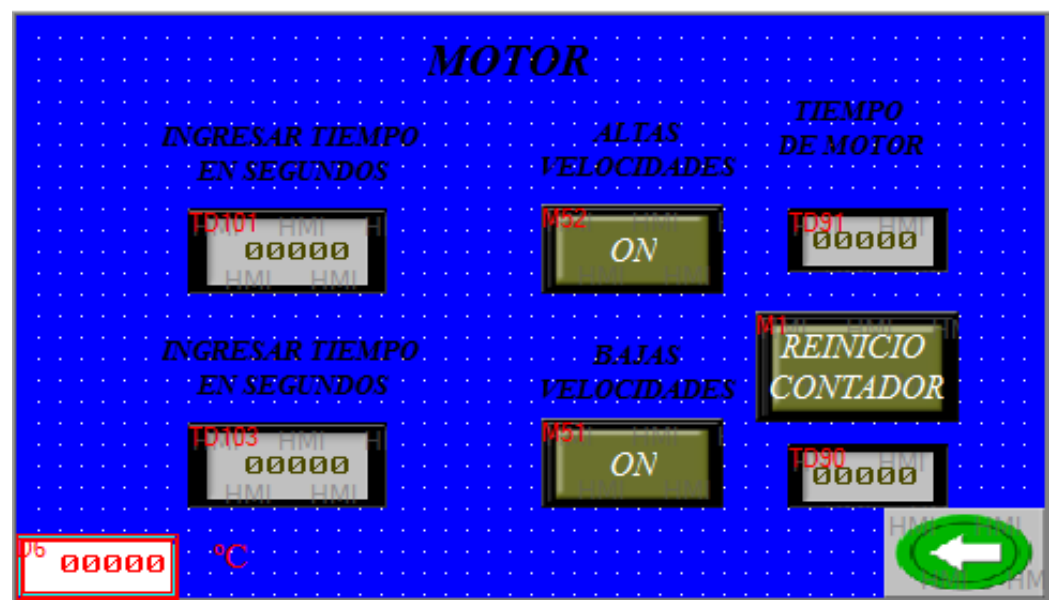

Figura 16-2 Ingreso tiempo de agitación Fuente: Elaboración propia 


\section{Procedimiento químico}

\section{Equipo de protección individual}

La utilización del equipo de protección individual permite reducir los riesgos que podrían amenazar la seguridad o salud del operador del equipo. En el Laboratorio de Procesos Industriales es obligatorio regirse por el Protocolo de Equipos de Protección Personal, el mismo que considera la utilización de:

- Protección Corporal (Mandil).

- Protección Visual (Gafas de seguridad).

- Protección Respiratoria (Mascarilla).

- Guantes de látex.

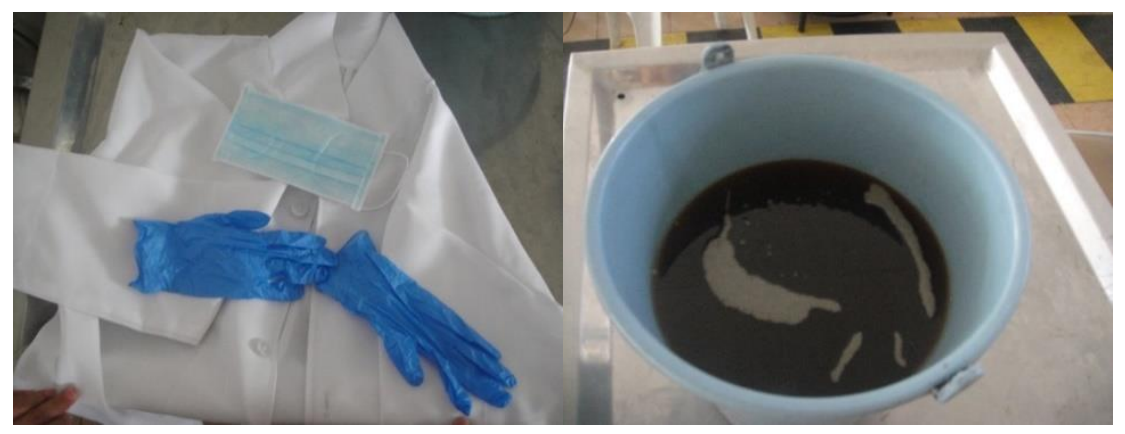

Figura 17-2 Equipo de protección individual empleado y aceite de fritura flitrado

Fuente: Elaboración propia

Limpieza del equipo

Antes y después de realizar las prácticas en el reactor, se debe verificar que se encuentre completamente limpio: el tanque de reacción, los tanques secundarios y el recipiente de descarga, caso contrario el resultado final puede no ser el esperado.

Preparación del reactivo y catalizador

\section{Metanol}

Se emplea el metanol absoluto, es decir, con una pureza del $99 \%$ para lograr una mejor conversión del aceite en biodiesel. Se emplea en proporción del $20 \%$ del volumen de aceite cargado.

\section{Hidróxido de sodio}

El hidróxido de sodio $(\mathrm{NaOH})$ se debe manejar con mucha precaución ya que es corrosivo y expuesto al ambiente absorbe la humedad y pierde propiedades químicas. Se necesita $3.5 \mathrm{~g}$ de hidróxido por cada litro de aceite cargado, se emplea una balanza para su pesaje.

\section{Mezcla de los reactivos}

La presentación del $\mathrm{NaOH}$ es en escamas y para agregarse al aceite debe estar completamente diluido; una vez pesada la cantidad de catalizador se coloca en el vaso de precipitación, se añade el metanol y se utiliza una barra de agitación para la mezcla. 
Esta preparación debe calentarse a $40^{\circ} \mathrm{C}$ por tiempos cortos en un reverbero eléctrico para diluir el $\mathrm{NaOH}$. La mezcla puede evaporarse y para evitar las pérdidas de metanol, el vaso de precipitación o el tanque secundario del metóxido debe permanecer cubierto con papel aluminio.
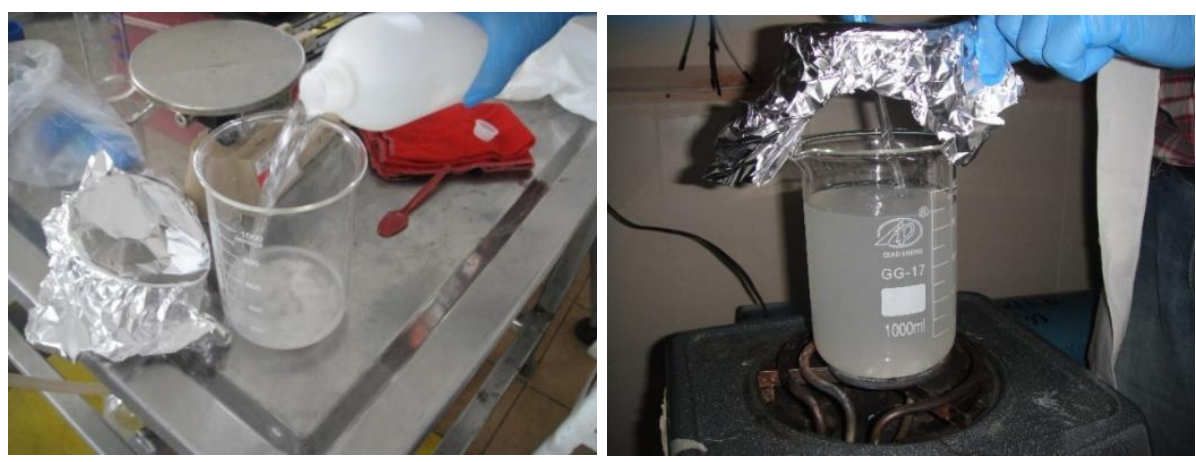

Figura 29-2 Mezcla Metóxido

Fuente: Elaboración propia

\section{Resultados.}

Para obtener los resultados se realizaron pruebas en el reactor de biodiesel antes de la intervención de la automatización para determinar los aspectos que provocaban ineficiencia en su operación y consumo excesivo de recursos. Posterior se realizó nuevas pruebas de funcionamiento para descartar cualquier tipo de fallo ya sea en hardware o software. Cabe recalcar que se realizaron cuadros comparativos de la funcionalidad del equipo previo y posterior a la automatización manteniendo las mismas condiciones de operación.

En el sistema de agitación antiguo estaba constituido por un taladro apostado sobre la tapa del reactor y sujetado por una base fija; además se debía mantener presionado el interruptor del taladro por una hora, lo que implicaba una variación en la velocidad y la presencia obligada del operario en esta parte del proceso.

Tabla 1-3: Manipulación antes y después de la automatización.

\begin{tabular}{lcc}
\hline \multicolumn{1}{c}{ Evento } & $\begin{array}{c}\text { Manipulación } \\
\text { Antes }\end{array}$ & $\begin{array}{c}\text { Manipulación } \\
\text { Después }\end{array}$ \\
\hline Carga de aceite / biodiesel & $\mathrm{Si}$ & $\mathrm{No}$ \\
Ingreso del metóxido & $\mathrm{Si}$ & $\mathrm{No}$ \\
Agitación en la fase de transesterificación & $\mathrm{Si}$ & $\mathrm{No}$ \\
Drenaje glicerina / agua & $\mathrm{Si}$ & $\mathrm{Si}$ \\
Ingreso de agua & $\mathrm{Si}$ & $\mathrm{No}$ \\
Agitación en la fase de lavado & $\mathrm{Si}$ & $\mathrm{No}$ \\
TOTAL $\boldsymbol{E} \boldsymbol{V E N T O S}$ & $\boldsymbol{6}$ & $\mathbf{5}$ \\
\hline
\end{tabular}

Fuente: Elaboración propia. 
En base a los resultados de la Tabla 1-3 el reactor en forma manual el operario debía estar presente y maniobrar el equipo en todas las etapas de la producción de biodiesel, con la automatización del equipo la intervención del usuario es necesario en una sola etapa debido a que el drenaje del agua o del glicerol se lo hace a través de las llaves de fondo. Debido a que la glicerina flote o se precipite depende de la calidad de la materia prima utilizada.

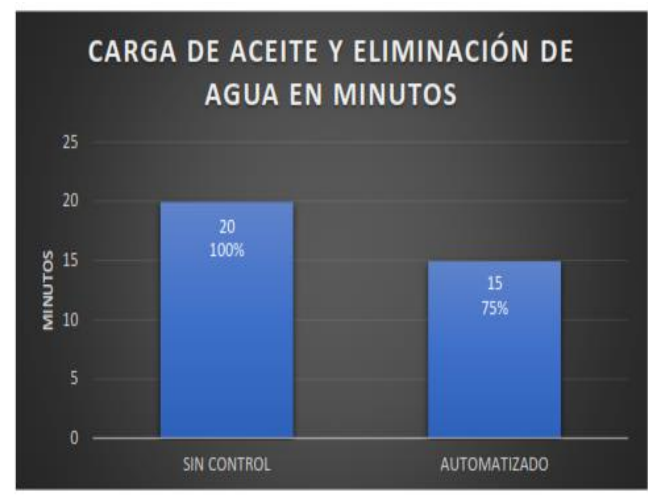

Gráfico 1-3 Tiempos en carga de aceite y eliminación de agua Realizado por: Campos J.y Rodriguez J.2016

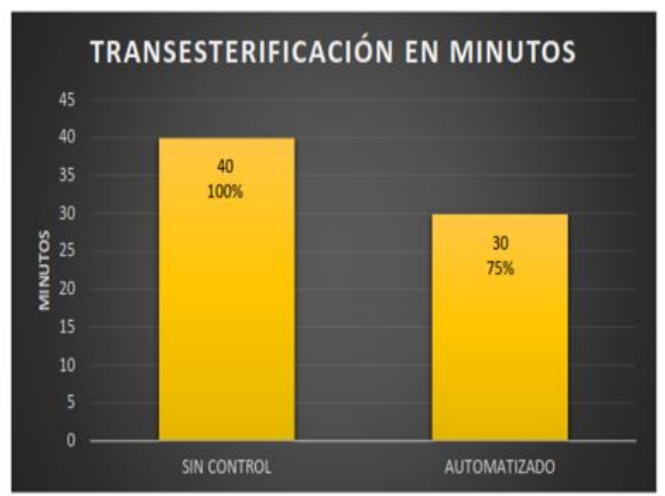

Gráfico 2-3 Tiempos en la fase de transesterificación Realizado por: Campos $J$. $y$ Rodrigucz 1.2016

La Figura 1-3 muestra la carga de aceite y eliminación de agua en minutos del reactor sin control y automatizado; se determinó que con el equipo automatizado se tiene una reducción del $25 \%$ del tiempo que tomaba hacerlo manualmente. En la Figura 2-3 corresponde a la fase de transesterificación la cual indica una reducción de 10 minutos, es decir el $25 \%$ de tiempo que tomaba al hacerlo de forma manual.

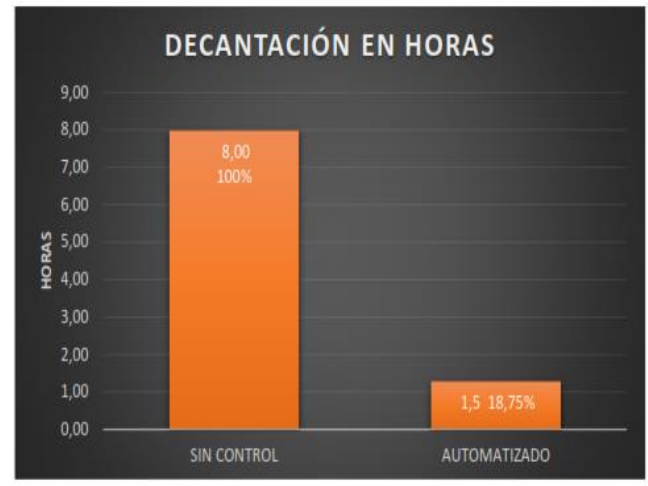

Gráfico 3-3 Tiempos en la fase de decantación Realizado por: Campos J. y Rodrigucz J. 2016

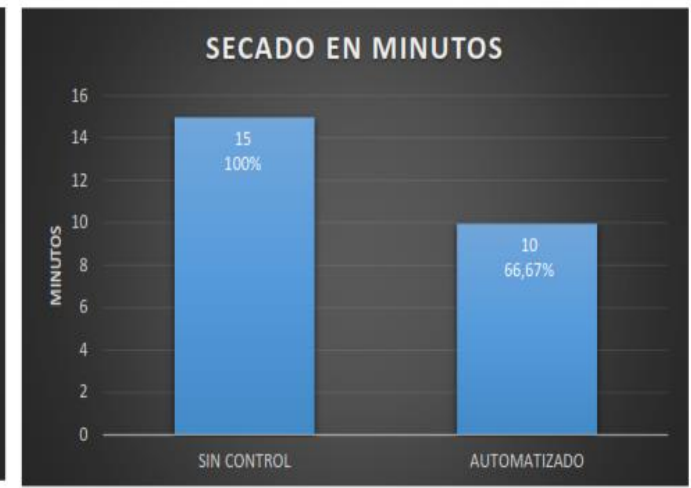

Gráfico 4-3 Tiempos en la fase de secado Realizado por: Campos J. y Rodriguce J. 2016

La Figura 3-3 muestra que la decantación en forma manual requería de un tiempo de 8 horas, al automatizar el tiempo se reduce a 1 hora y 30 minutos lo que representa una reducción del $81,25 \%$ del tiempo en la fase de decantación. La Figura 4-3 se puede observar que la fase de secado se lleva a cabo en 10 minutos, hasta alcanzar el punto de ebullición del agua, lo que representa una reducción del tiempo en un 33.33\% comparado a la operación en forma manual.

\section{Análisis y comparaciones de tiempos del proceso completo}

En la automatización del reactor de biodiesel se obtuvieron datos de tiempos y temperaturas, en base a cargas de 10 litros de aceite de cocina reciclado, teniendo en 
cuenta que la llave de paso de vapor desde la caldera debía estar abierta a la mitad y trabajar con una presión mínima de 40 PSI y máxima de 50 PSI.

Tabla 2-3: Tiempos del reactor en operación manual

\begin{tabular}{|c|c|c|}
\hline Proceso / Fase & Tiempo & Temperatura \\
\hline Carga de aceite & $5 \min$ & Ambiente \\
\hline Eliminación de agua & $15 \min$ & $100^{\circ} \mathrm{C}$ \\
\hline Tiempo de espera hasta llegar a $60^{\circ} \mathrm{C}$ & $1 \mathrm{~h} 30 \mathrm{~min}$ & \\
\hline Transesterificación & $40 \mathrm{~min}$ & $57^{\circ} \mathrm{C} \pm 3^{\circ} \mathrm{C}$ \\
\hline Decantación & $8 \mathrm{~h}$ & Ambiente \\
\hline Drenaje de la glicerina & $5 \mathrm{~min}$ & Ambiente \\
\hline Lavado del biodiesel & $15 \mathrm{~min}$ & Ambiente \\
\hline Drenaje de agua & $5 \mathrm{~min}$ & Ambiente \\
\hline Tiempo de reposo para separar los jabones & $20 \min$ & Ambiente \\
\hline Secado & $15 \min$ & $100^{\circ} \mathrm{C}$ \\
\hline TOTAL TIEMPO & $11 \mathrm{~h} 30 \mathrm{~min}$ & \\
\hline
\end{tabular}

Fuente: Elaboración propia.

De acuerdo a la Tabla 2-3 el reactor operaba en manera manual con un tiempo de 11 horas y media, la etapa que más tiempo consumía era la de decantación con un tiempo de 8 horas.

Tabla 3-3: Tiempos del reactor automatizado

\begin{tabular}{lll}
\hline \multicolumn{1}{c}{ Proceso / Fase } & \multicolumn{1}{c}{ Tiempo } & Temperatura \\
\hline Carga de aceite y eliminación de agua & $15 \mathrm{~min}$ & $100^{\circ} \mathrm{C}$ \\
$\begin{array}{l}\text { Tiempo de espera hasta que llegue a } 60^{\circ} \mathrm{C} \text { (fuera del } \\
\text { reactor) }\end{array}$ & $50 \mathrm{~min}$ & Ambiente \\
Transesterificación & $30 \mathrm{~min}$ & $58^{\circ} \mathrm{C} \pm 2^{\circ} \mathrm{C}$ \\
Decantación & $1 \mathrm{~h} 30 \mathrm{~min}$ & $>38^{\circ} \mathrm{C}$ \\
Drenaje de la glicerina & $5 \mathrm{~min}$ & Ambiente \\
Lavado del biodiesel & $15 \mathrm{~min}$ & Ambiente \\
Drenaje de agua & $5 \mathrm{~min}$ & Ambiente \\
Tiempo de reposo para separar los jabones & $20 \mathrm{~min}$ & Ambiente \\
Secado & $10 \mathrm{~min}$ & $100^{\circ} \mathrm{C}$ \\
TOTAL TIEMPO & $\mathbf{4 h}$ &
\end{tabular}

Fuente: Elaboración propia. 
En la Tabla 3-3 muestra los resultados obtenidos del reactor automatizado con un total de tiempo de 4 horas, considerando que las etapas de drenaje y glicerina se realizan de manera manual debido a las características del aceite reciclado hacen que la glicerina se precipite al fondo del reactor o flote sobre el biodiesel.

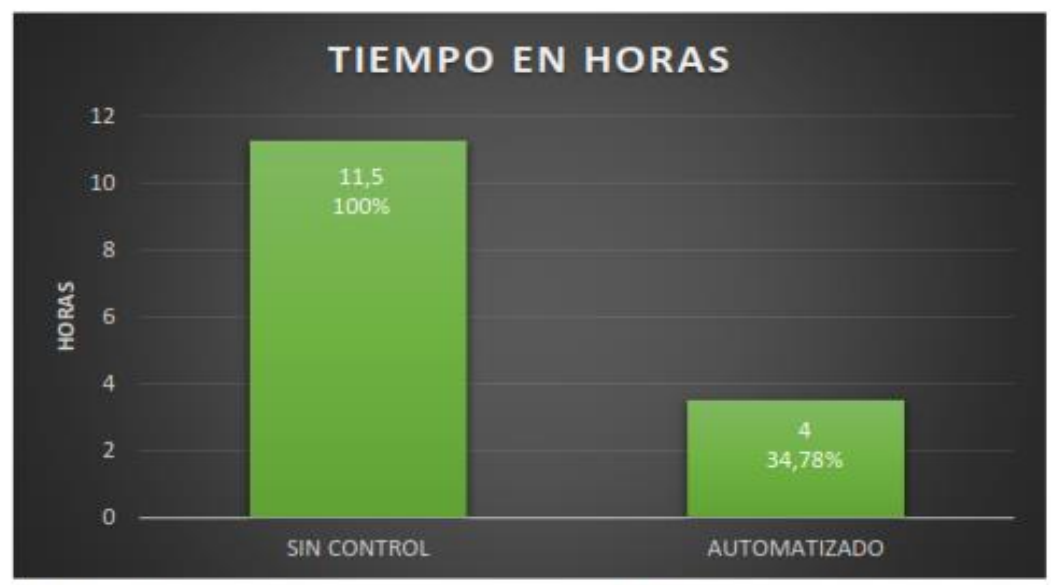

Gráfico 5-3 Tiempo en la producción del biodiesel Realizado por: Campos J. y Rodriguez J. 2016

En el Grafico 5-3 muestra los resultados obtenidos, en forma manual para la obtención del biodiesel el equipo necesitaba alrededor de 11 horas y 30 minutos, con la automatización se llevó a cabo el mismo proceso en solo 4 horas, lo que representa una disminución del 65,22\% del tiempo inicial.

\section{Rendimiento del reactor automatizado}

Una vez automatizado el reactor se realizó evaluaciones de consumo y rendimiento, cabe indicar que el reactor no automatizado presentaba un consumo de 2,5 A el cual correspondía a la corriente que consumía el taladro, en la automatización se incorporó dispositivos eléctricos por lo tanto se obtuvo un consumo aproximado de 4 A el cual sigue siendo un valor bajo este aumento de corriente se debe al consumo de la bomba de agua que presenta una corriente nominal de 3,3 A.

El rendimiento se calculó en base al número de horas que se empleaba el reactor de manera manual sobre el tiempo que realiza el mismo proceso de manera automático y dio como resultado el $287,5 \%$ de rendimiento.

\section{Conclusiones.}

- Se establecieron las características y parámetros para el funcionamiento y operación del reactor, determinando: fases de producción, valores de temperatura, tiempos, velocidades de agitación y cantidades de reactivos químicos.

- El PLC permite el accionamiento de los actuadores en función de las señales recibidas y las condiciones programadas, enlazándose al HMI para facilitar el control y monitoreo del equipo. 
- La decantación es una de las fases de producción del biodiesel, crítica debido al tiempo que se requería para llevarla a cabo de forma manual (8 horas mínimo). La forma de reducir el tiempo en esta fase es manteniendo la mezcla en reposo, después de la reacción, y la temperatura sobre $\operatorname{los} 38^{\circ} \mathrm{C}$ por una hora y media. Se logra reducir el tiempo en un $81,25 \%$.

- El controlador, los sensores y actuadores se seleccionaron según los requerimientos del proceso; los conductores eléctricos, fuente de $\mathrm{CC}$, y la protección se determinaron en función de la carga instalada.

- El rendimiento del reactor automatizado se incrementa notablemente en $287,5 \%$. Se tiene un equipo operativo y seguro, muestra bajo consumo de corriente, reducción en la cantidad de agua empleada para el lavado del biodiesel y posee un sistema de agitación constante. El rendimiento en volumen del biodiesel obtenido depende en gran medida de la calidad del aceite de cocina reciclado y la pureza del metanol.

\section{Referencias bibliográficas.}

ARIAN, Arian Control \& Instrumentación. Pt100, su operación, instalación y tablas. [En línea] 2021. [Consulta: 28 Marzo 2021]. Disponible en: http://www.arian.cl/downloads/nt-004.pdf

CAMPOS, Jonatahn: RODRIGUEZ, Juan. Automatización de un reactor tipo batch para la obtención de biodiesel en el Laboratorio de Procesos Industriales de la Facultad de Ciencias. [En línea] 2021. [Consulta: 28 Mayo 2021]. Disponible en: http://dspace.espoch.edu.ec/handle/123456789/6121

ELECTRONICA, Electrónica Fácil. Fuentes de Alimentación. [En línea] 2016. [Consulta: 29 Marzo 2021]. Disponible en: http://www.electronicafacil.net/tutoriales/Fuentes-alimentacion.php

PALMAY, Paúl. Diseño y construcción de un reactor batch para la obtención por transesterificación de biodiesel a partir de aceite de cocina reciclado [En línea] (Tesis Pregrado) ESPOCH, Ciencias, Ingeniería Química, Riobamba-Ecuador. 2009. pp. 35-80. [Consulta: 1 Marzo 2021]. Disponible en: http://dspace.espoch.edu.ec/handle/123456789/248.pdf

UNICROM, Electrónica Unicrom. Relé - Relay - Relevador (Interruptor operado magnéticamente). [En línea] 2021. [Consulta: 28 Marzo 2021]. Disponible en: http://unicrom.com/rele-relay-relevador-interruptor-operado-magneticamente/

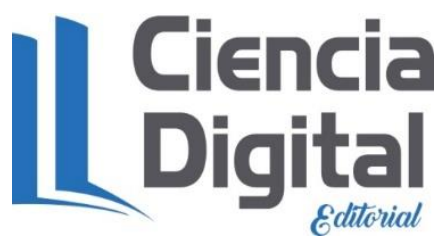




\section{PARA CITAR EL ARTÍCULO INDEXADO.}

Campos Castelo, J. P., Coba Castillo, N. J., Villa Escudero, I. C., \& Aldaz Vargas, A. A. (2021). Automatización de un Reactor Tipo Batch para la obtención de Biodiesel a partir de aceite reciclado. AlfaPublicaciones, 3(3), 57-77. https://doi.org/10.33262/ap.v3i3.68

\section{Ciencia \\ LDigital}

El artículo que se publica es de exclusiva responsabilidad de los autores y no necesariamente reflejan el pensamiento de la Revista Alfa Publicaciones.

El artículo queda en propiedad de la revista y, por tanto, su publicación parcial y/o total en otro medio tiene que ser autorizado por el director de la Revista Alfa Publicaciones.
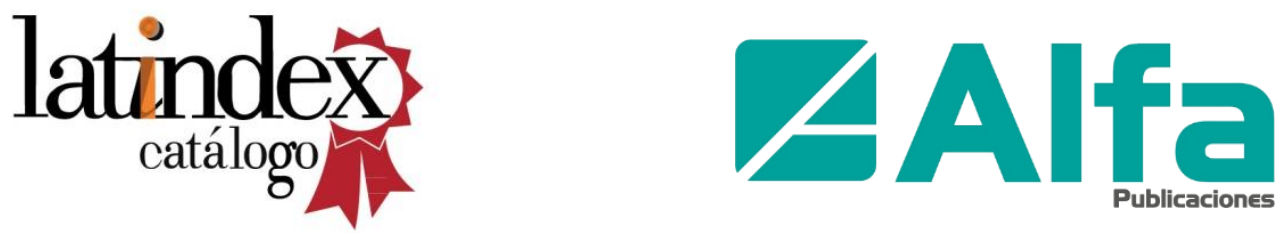